\title{
THE EQUIVALENCE OF SEQUENCE INTEGRALS AND NON-ABSOLUTELY CONVERGENT INTEGRALS*
}

\author{
R. L. JEFFERY
}

This note completes and extends some results previously obtained. $\dagger$ Let the function $f(x)$ be measurable, and finite almost everywhere on $(a, b)$. Let $s_{n}(x)$ be a sequence of summable functions such that $s_{n}=f$ on a set $E_{n}, s_{n}=0$ elsewhere, $E_{n} \supset E_{n-1}$, and $m E_{n} \rightarrow b-a$. If $\int_{a}^{x} s_{n} d x$ tends to a continuous function $\phi(x)$, then $f$ is, by definition, totally integrable in the sequence sense to $\phi(x)=T S(f, a, x)$. It has been shown that if $f(x)$ is integrable in the generalized Denjoy sense to $F(x)=\int_{a}^{x} f(x) d x$, then there exists $T S(f, a, x)=F(x)$. Such a function $T S(f, a, x)$ is generalized absolutely continuous (ACG), $\S$ since $F(x)$ is (ACG). A function $T S(f, a, x)$ was constructed $\|$ which was not (ACG) and consequently not equal to $F(x)$. This raised the question as to whether or not the property of being (ACG) was sufficient to insure that $T S(f, a, x)=F(x)$. In the present note this question is answered in the negative, and necessary and sufficient conditions are determined for the relation $T S(f, a, x)=F(x)$.

We first construct a function $f(x)$ which is not summable, but which is integrable in a non-absolutely convergent sense, and then construct $T S(f, a, x)$ which is (ACG) and not equal to $F(x)=\int_{a}^{x} f d x$. Let $G$ be a Cantor set on $(a, b)$ with $m G>0$, and let $\left(\alpha_{i}, \beta_{i}\right)$ be the intervals complementary to $G$. On $\left(\alpha_{i}, \beta_{i}\right)$ construct $f_{i}$ such that $\int_{\alpha_{i}}^{\beta_{i}} f_{i} d x$ exists as a non-absolutely convergent integral with $\beta_{i}$ the single point of nonsummability of $f_{i}$, with $\int_{\alpha_{i}}^{\beta_{i}} f_{i} d x=0$, and with $\left|\int_{\alpha_{i}}^{x} f_{i} d x\right|<\beta_{i}-\alpha_{i}$ for $x$ on $\left(\alpha_{i}, \beta_{i}\right)$. Let $f(x)=f_{i}(x)$ on $\left(\alpha_{i}, \beta_{i}\right)$, and $f(x)=0$ elsewhere. Then $F(x)=\int_{a}^{x} f d x$ exists as a non-absolutely convergent integral, and $F(x)=0$ for $x$ a point of $G$. Consider the set of intervals $\left(\alpha_{i}, \beta_{i}\right)$ ordered in any way. Then take the first $n$ intervals of this set and order them from left to right into the set $\left(\alpha_{1}^{\prime}, \beta_{1}^{\prime}\right), \cdots,\left(\alpha_{n}^{\prime}, \beta_{n}^{\prime}\right)$. To the right of each interval $\left(\alpha_{j}^{\prime}, \beta_{j}^{\prime}\right)$ there is an interval $\lambda_{n i}=\left(\beta_{j}^{\prime}, \alpha_{j+1}^{\prime}\right)$, where $i$ is the subscript that $\left(\alpha_{i}, \beta_{i}\right)$ has in the original ordering

* Presented to the Society, January 1, 1936.

$\dagger$ Transactions of this Society, vol. 41 (1935), pp. 171-192. In what follows this paper will be referred to as $T$.

$\ddagger$ T, p. 186, Theorem 6 .

§ Saks, Thêorie de l'Intégrale, Warsaw, 1933, p. 152, §9.

\| T, pp. 189-191. 
$\left(\alpha_{i}, \beta_{i}\right)$. On $\left(\alpha_{j}^{\prime}, \beta_{j}^{\prime}\right)$ fix a set $E_{n i}$ such that if $s_{n i}=f$ on $E_{n i}$ and $s_{n i}=0$ elsewhere, then

$$
\left|\int_{\alpha_{j}^{\prime}}^{\beta_{j^{\prime}}} s_{n i} d x-m \lambda_{n i}\right|<\frac{1}{n^{2}} .
$$

If $s_{n}=s_{n i}$ on $\left(\alpha_{j}^{\prime}, \beta_{j}^{\prime}\right)$ and $s_{n}=0$ elsewhere, then

$$
\left|\int_{a}^{b} s_{n} d x-\sum m \lambda_{n i}\right|<\frac{1}{n} \text {. }
$$

As $n$ increases it is possible to choose the sets $E_{n i}$ in such a way that $E_{(n+1) i} \supset E_{n i}$ and $m E_{n i} \rightarrow \beta_{i}-\alpha_{i}$. If this is done, it is then easily verified that

$$
\lim _{n \rightarrow \infty} \int_{a}^{x} s_{n} d x=F(x)+m G(a, x)=T S(f, a, x),
$$

where $G(a, x)$ is the part of $G$ on $(a, x)$. The function $F(x)$ is (ACG), and the function $m G(a, x)$ is (AC). Hence $T S(f, a, x)$ is (ACG). Furthermore, since $m G>0$, we have $T S(f, a, x) \neq F(x)$.

The foregoing considerations lead us to seek necessary and sufficient conditions that $T S(f, a, x)=F(x)$. Associated with the function $\phi(x)=T S(f, a, x)$ is a function of sets $\phi(G)=\lim \int_{G} s_{n} d x$, provided this limit exists, where $G$ is a measurable set on $(a, b)$ and $s_{n}$ is the sequence involved in the definition of $T S(f, a, x)$. If $G$ is an interval $(\alpha, \beta)$, then $\phi(\alpha, \beta)=T S(f, \alpha, \beta)$. The function of sets $\phi(G)$ is completely additive if, for every set of disjunct sets $G_{1}, G_{2}, \cdots$, we have the relation $\phi\left(\sum G_{i}\right)=\sum \phi\left(G_{i}\right)$. The function $\phi(G)$, associated with the function $f(x)$ defined above, is not completely additive. For if $\left(\alpha_{i}, \beta_{i}\right)$ is the set of open intervals complementary to the set $E$, then $\phi\left[\sum\left(\alpha_{i}, \beta_{i}\right)\right]=m E$, while $\sum \phi\left(\alpha_{i}, \beta_{i}\right)=0$. We prove the following theorem:

Theorem 1. If $f(x)$ is integrable in the generalized Denjoy sense to $F(x)$, and if $\phi(x)=T S(f, a, x)$ is such that $\phi(G)$ is completely additive, then $\phi(x)=F(x)$.

Let $E_{1}$ be the points of non-summability of $f$ over $(a, b),\left(\alpha_{i}, \beta_{i}\right)$ the intervals on $(a, b)$ complementary to $E_{1}$, and $\left(\alpha_{i}^{\prime}, \beta_{i}^{\prime}\right)$ an interval with $\alpha_{i}<\alpha_{i}^{\prime}<\beta_{i}^{\prime}<\beta_{i}$. The function $f$ is summable on $\left(\alpha_{i}^{\prime}, \beta_{i}^{\prime}\right)$, and

$$
\phi\left(\beta_{i}^{\prime}\right)-\phi\left(\alpha_{i}^{\prime}\right)=\int_{\alpha_{i^{\prime}}}^{\beta_{i^{\prime}}} f d x=F\left(\beta_{i}^{\prime}\right)-F\left(\alpha_{i}^{\prime}\right) .
$$

It then follows from the continuity of $F$ and $\phi$ that $\phi\left(\beta_{i}\right)-\phi\left(\alpha_{i}\right)$ $=F\left(\beta_{i}\right)-F\left(\alpha_{i}\right)$. Let $E_{2}$ be the points of non-summability of $f$ over $E_{1}$ 
together with the points of $E_{1}$ at which $\sum\left|F\left(\beta_{i}\right)-F\left(\alpha_{i}\right)\right|$ diverges, let $\left(\alpha_{j}, \beta_{j}\right)$ be the intervals on $(a, b)$ complementary to $E_{2}$, and let $\left(\alpha_{j}^{\prime}, \beta_{j}^{\prime}\right)$ be an interval with $\alpha_{j}<\alpha_{j}^{\prime}<\beta_{j}^{\prime}<\beta_{j}$. Then

$$
\phi\left(\beta_{j}^{\prime}\right)-\phi\left(\alpha_{j}^{\prime}\right)=\lim _{n \rightarrow \infty} \int_{\alpha_{j^{\prime}}}^{\beta_{j^{\prime}}} s_{n} d x=\lim _{n \rightarrow \infty} \int_{\Sigma\left(\alpha_{l}, \beta_{l}\right)} s_{n} d x+\lim _{n \rightarrow \infty} \int_{e} s_{n} d x,
$$

where $e$ is the part of $E_{1}$ on $\left(\alpha_{j}^{\prime}, \beta_{j}^{\prime}\right)$, and $\sum\left(\alpha_{l}, \beta_{l}\right)$ is the part of the set $\left(\alpha_{i}, \beta_{i}\right)$ on $\left(\alpha_{j}^{\prime}, \beta_{j}^{\prime}\right)$. The second limit on the right exists for the reason that $f$ is summable over $e$. Consequently the first limit on the right exists. Then, since $\phi(G)$ is completely additive,

$$
\begin{aligned}
\phi\left(\beta_{j}^{\prime}\right)-\phi\left(\alpha_{j}^{\prime}\right) & =\sum \lim _{l} \int_{\alpha_{l}}^{\beta_{l}} s_{n} d x+\int_{e} f d x \\
& =\sum\left\{\phi\left(\beta_{l}\right)-\phi\left(\alpha_{l}\right)\right\}+\int_{e} f d x \\
& =\sum\left\{F\left(\beta_{l}\right)-F\left(\alpha_{l}\right)\right\}+\int_{e} f d x \\
& =F\left(\beta_{j}^{\prime}\right)-F\left(\alpha_{j}^{\prime}\right) .
\end{aligned}
$$

Again the continuity of $F$ and $\phi$ gives $\phi\left(\beta_{j}\right)-\phi\left(\alpha_{j}\right)=F\left(\beta_{j}\right)-F\left(\alpha_{j}\right)$. This process can be continued by means of finite and transfinite induction to give $\phi(x)=F(x)$ for $x$ on $(a, b)$.

The complete additivity of $\phi(G)$ is not a necessary condition that $\phi(x)=T S(f, a, x)=F(x)$. Let $x_{0}=0<x_{1}<x_{2}<\cdots$ be a sequence of values of $x$ on $(0,1)$ with $x_{n}$ tending to unity. Let $f$ be so defined on $\left(x_{n-1}, x_{n}\right)$ that the integral of $f$ over this interval exists as a nonabsolutely convergent integral with $x_{n}$ the single point of nonsummability. Furthermore, let $f$ be such that if $F(x)=\int_{a}^{x} f d x$, then $F\left(x_{n}\right)-F\left(x_{n-1}\right)=(-1)^{n-1} n$. There then exists $T S(f, a, x)^{*}$ such that

$$
\phi(x)=T S(f, a, x)=\lim _{n \rightarrow \infty} \int_{0}^{x} s_{n} d x=F(x) .
$$

The set $E$ of points of non-summability of $f$ is the set $x_{0}, x_{1}, \cdots$, and unity. Let $\left(\alpha_{i}, \beta_{i}\right)$ be the intervals complementary to $E$. We have

$$
\begin{aligned}
F(1)-F(0) & =\lim _{n \rightarrow \infty} \int_{0}^{1} s_{n} d x=\lim _{n \rightarrow \infty} \int_{E} s_{n} d x+\lim _{n \rightarrow \infty} \int_{C E} s_{n} d x \\
& =\lim _{n \rightarrow \infty} \int_{\Sigma\left(\alpha_{i}, \beta_{i}\right)} s_{n} d x=\phi\left[\sum\left(\alpha_{i}, \beta_{i}\right)\right] .
\end{aligned}
$$

* T, p. 186, Theorem 6. 
But the value of $\sum \phi\left(\alpha_{i}, \beta_{i}\right)$ depends on the order in which the intervals $\left(\alpha_{i}, \beta_{i}\right)$ are taken.

TheOREM 2. Let $F(x)=\int_{a}^{x} f d x$, where the integration is in the generalized Denjoy sense. Let $\phi(x)=T S(f, a, x)$. A necessary and sufficient condition that $\phi(x)=F(x)$ is that if $(l, m)$ is an interval on $(a, b)$ containing a closed set e over which $f$ is summable, if $\left(\alpha_{i}, \beta_{i}\right)$ are the intervals on $(l, m)$ contiguous to $e$, and if $\sum\left|\phi\left(\beta_{i}\right)-\phi\left(\alpha_{i}\right)\right|$ converges, then $\phi\left[\sum\left(\alpha_{i}, \beta_{i}\right)\right]=\sum \phi\left(\alpha_{i}, \beta_{i}\right)$.

The proof of the sufficiency of the conditions follows the same lines as the proof of Theorem 1 . To show that the conditions are necessary let $\phi(x)=T S(f, a, x)=F(x)$. Then

$$
\begin{aligned}
\phi(m)-\phi(l) & =\lim _{n \rightarrow \infty} \int_{\Sigma\left(\alpha_{i}, \beta_{i}\right)} s_{n} d x+\lim _{n \rightarrow \infty} \int_{e} s_{n} d x \\
& =\phi\left[\sum\left(\alpha_{i}, \beta_{i}\right)\right]+\int_{e} f d x .
\end{aligned}
$$

From this, and the fact that $\phi(x)=F(x)$, it follows that $\phi\left[\sum\left(\alpha_{i}, \beta_{i}\right)\right]$ $=\sum\left\{F\left(\beta_{i}\right)-F\left(\alpha_{i}\right)\right\}=\sum\left\{\phi\left(\beta_{i}\right)-\phi\left(\alpha_{i}\right)\right\}$.

In the foregoing $f$ is summable over $e$. Suppose that $f$ is not summable over $e$, but that $\int_{e} f d x$ exists as a non-absolutely convergent integral, and suppose that $\sum\left|\phi\left(\beta_{i}\right)-\phi\left(\alpha_{i}\right)\right|$ converges. Is it necessary that $\phi\left[\sum\left(\alpha_{i}, \beta_{i}\right)\right]=\sum \phi\left(\alpha_{i}, \beta_{i}\right)$ ? We answer this question in the negative. Returning to the first example above, we bisect the intervals $\left(\alpha_{i}, \beta_{i}\right)$, getting the intervals $\left(\alpha_{i}, a_{i}\right),\left(a_{i}, \beta_{i}\right)$. On each of these intervals we define $f_{i}$ in the way that $f_{i}$ was defined on the original interval $\left(\alpha_{i}, \beta_{i}\right)$. In particular, $a_{i}$ will be the single point of non-summability of $f_{i}$ on $\left(\alpha_{i}, a_{i}\right)$, and $\beta_{i}$ will be the single point of non-summability of $f_{i}$ on $\left(a_{i}, \beta_{i}\right)$. Let $f=f_{i}$ on $\left(\alpha_{i}, a_{i}\right)$ and on $\left(a_{i}, \beta_{i}\right)$, and let $f=0$ elsewhere. Going to the interval $\left(\alpha_{j}^{\prime}, \beta_{j}^{\prime}\right)$ we determine sets $E_{n i}^{\prime \prime}$ on $\left(\alpha_{j}^{\prime}, a_{i}\right)$ and $E_{n i}^{\prime \prime}$ on $\left(a_{i}, \beta_{j}^{\prime}\right)$ in such a way that if $s_{n}^{\prime}=f$ on $E_{n i}^{\prime \prime}$, and $s_{n}=0$ elsewhere, then $\int_{a}^{x} s_{n} d x \rightarrow m G(a, x)$; if $s_{n}^{\prime \prime}=f$ on $E_{n i}^{\prime \prime}$ and $s_{n}^{\prime \prime}=0$ elsewhere, then $\int_{a}^{x} s_{n} d x \rightarrow-m G(a, x)$. If further $s_{n}=s_{n}{ }^{\prime}+s_{n}{ }^{\prime \prime}$, then

$$
\phi(x)=\lim _{n \rightarrow \infty} \int_{a}^{x} s_{n} d x=T S(f, a, x)=\int_{a}^{x} f d x=F(x) .
$$

Let $e$ be the closed set complementary to the set of open intervals $\left(\alpha_{i}, a_{i}\right)$. Then $\int_{e} f d x$ exists as a non-absolutely convergent integral. But $\phi\left[\sum\left(\alpha_{i}, a_{i}\right)\right]=m G$, and $\sum \phi\left(\alpha_{i}, a_{i}\right)=0$. It thus appears that in so far as it is a question of complete additivity, the conditions of Theorem 2 are the best possible. 
So far it has been assumed that $f(x)$ is integrable in a non-absolutely convergent sense. We now prove the following theorem:

THEOREM 3. Let the function $f(x)$ be measurable on $(a, b)$, and let $T S(f, a, x)$ exist. If $E$ is any closed set on $(a, b)$, let an interval $(l, m)$ containing a part e of $E$ exist such that $f$ is summable over e, $\sum\left|\phi\left(\alpha_{i}, \beta_{i}\right)\right|$ converges, and $\sum \phi\left(\alpha_{i}, \beta_{i}\right)=\phi\left[\sum\left(\alpha_{i}, \beta_{i}\right)\right]$, where $\left(\alpha_{i}, \beta_{i}\right)$ are the intervals complementary to $e$ on $(l, m)$. Then $f$ is integrable in the generalized Denjoy sense, and $\int_{a}^{x} f d x=T S(f, a, x)$.

If the closed set $E$ of the theorem is the interval $(a, b)$, then the part $e$ of $E$ on $(l, m)$ is all of $(l, m)$, and it follows that the points $E_{1}$ of non-summability of $f$ over $(a, b)$ are non-dense on $(a, b)$. Let $(\alpha, \beta)$ be an interval complementary to $E_{1}$. The function $f$ is then summable over any interval interior to $(\alpha, \beta)$, and as a consequence of this $\phi^{\prime}=f$ almost everywhere on $(\alpha, \beta)$. The set $E_{1}$ is closed. By the conditions of the theorem there is an interval $(l, m)$ containing a part $e$ of $E_{1}$ with $f$ summable over $e, \sum\left|\phi\left(\alpha_{i}, \beta_{i}\right)\right|$ convergent, and $\sum \phi\left(\alpha_{i}, \beta_{i}\right)$ $=\phi\left[\sum\left(\alpha_{i}, \beta_{i}\right)\right]$. For $x$ on this interval $(l, m)$, we have

$$
\begin{aligned}
\phi(x)-\phi(l) & =\lim _{n \rightarrow \infty} \int_{l}^{x} s_{n} d x \\
& =\lim _{n \rightarrow \infty} \int_{e(l, x)} s_{n} d x+\lim _{n \rightarrow \infty} \int_{\Sigma_{(l, x)}\left(\alpha_{i}, \beta_{i}\right)} s_{n} d x \\
& =\int_{e} f d x+\sum_{(l, x)} \phi\left(\alpha_{i}, \beta_{i}\right)+\phi\left(\alpha_{k}, x\right)
\end{aligned}
$$

where the second term on the right represents the whole intervals of the set $\left(\alpha_{i}, \beta_{i}\right)$ on $(l, x)$, and the third term is absent unless $x$ is an interior point of an interval $\left(\alpha_{k}, \beta_{k}\right)$ of the set $\left(\alpha_{i}, \beta_{i}\right)$. Set $\psi(x)=\phi_{1}(x)+\phi_{2}(x)$, where

$$
\phi_{1}=\int_{e(l, x)} f d x, \quad \phi_{2}=\sum_{(l, x)} \phi\left(\alpha_{i}, \beta_{i}\right) .
$$

The function $\psi$ is constant on $\left(\alpha_{i}, \beta_{i}\right)$ and equal to $\phi$ at points of $e$. Furthermore, $\phi_{1}^{\prime}=f$ at almost all of $e$, and $\phi_{2}^{\prime}=0$ at almost all of $e$. $^{*}$ Consequently $\psi^{\prime}=f$ almost everywhere on $e$, and since $\psi=\phi$ at points of $e$, it follows that $\phi$ has an approximate derivative equal to $f$ at almost all points of $e$. If now $E_{2}$ denotes the points of $E_{1}$ which are points of non-summability of $f$ over $E$, or the points of $E_{1}$ at

\footnotetext{
* Denjoy, Journal de Mathématique, (7), vol. 1, p. $158 \mathrm{ff}$.
} 
which $\sum\left|\phi\left(\alpha_{i}, \beta_{i}\right)\right|$ either diverges or converges with $\sum \phi\left(\alpha_{i}, \beta_{i}\right)$ $\neq \phi\left[\sum\left(\alpha_{i}, \beta_{i}\right)\right]$, then the conditions of the theorem and the above reasoning allow us to conclude that the set $E_{2}$ is non-dense on $E_{1}$, and that if $(\alpha, \beta)$ is an interval of the set complementary to $E_{2}$, then $\phi$ has an approximate derivative equal to $f$ at almost all points of this interval. This process can be continued by finite and transfinite induction to show that $\phi$ has an approximate derivative equal to $f$ almost everywhere on $(a, b)$.

It will next be shown that $\phi(x)=T S(f, a, x)$ is (ACG). It is sufficient to show that for every closed set $E$ there exists an interval containing a part $e$ of $E$ over which $\phi$ is absolutely continuous.* Let $E$ be any closed set on $(a, b)$, and $(l, m)$ an interval containing a part $e$ of $E$ over which $f$ is summable and for which $\sum\left|\phi\left(\alpha_{i}, \beta_{i}\right)\right|$ converges with $\sum \phi\left(\alpha_{i}, \beta_{i}\right)=\phi\left[\sum\left(\alpha_{i}, \beta_{i}\right)\right]$. Let $\left(a^{\prime}, b^{\prime}\right)$ be an interval on $(l, m)$ with $a^{\prime}, b^{\prime}$ points of $e$. Then

$$
\left|\phi\left(b^{\prime}\right)-\phi\left(a^{\prime}\right)\right|=\left|\int_{e\left(a^{\prime}, b^{\prime}\right)} f d x+\sum_{\left(a^{\prime}, b^{\prime}\right)} \phi\left(\alpha_{i}, \beta_{i}\right)\right| .
$$

Since $\sum \phi\left(\alpha_{i}, \beta_{i}\right)$ converges, it is clear that the right-hand side of this equation can be made arbitrarily small by taking $b^{\prime}-a^{\prime}$ sufficiently small. Similarily, $\sum\left|\phi\left(b_{k}^{\prime}\right)-\phi\left(a_{k}^{\prime}\right)\right|$ is arbitrarily small if $\left(a_{k}^{\prime}, b_{k}^{\prime}\right)$ is a finite or denumerably infinite set of intervals of $(l, m)$ with $a_{k}^{\prime}, b_{k}^{\prime}$ points of $e$ and $\sum\left(b_{k}^{\prime}-a_{k}^{\prime}\right)$ sufficiently small. But this means that $\phi$ is (AC) on $e$ and consequently (ACG) on $(a, b)$.

We now have $\phi(x)$ (ACG) on $(a, b)$ and the approximate derivative of $\phi$ equal to $f$ almost everywhere. This allows us to conclude that $f$ is integrable in the generalized Denjoy sense, $\uparrow$ and that

$$
\phi(x)=\int_{a}^{x} f d x=T S(f, a, x) .
$$

The UnIVERSITY OF Wisconsin

* Saks, loc. cit., p. 165.

† Saks, loc. cit., p. 197, §2. 